\title{
"Jóvenes con más y mejor trabajo": desarrollo de sociabilidad grupal juvenil como efecto (inesperado) en la implementación de política pública destinada a jóvenes en Argentina*
}

\author{
"Youth with more and better jobs": developing young \\ group sociability as an effect (unexpected) in the \\ implementation of the public policy aimed at young \\ people in Argentina
}

\begin{abstract}
"Jovens com mais e melhor trabalho": desenvolvimento de sociabilidade grupal juvenil como efeito (inesperado) na implementação de política publica destinada a jovens
\end{abstract} na Argentina

\section{Andrea Bonvillani** - Argentina}

Recibido el 24 de marzo de 2012, aceptado el 17 de diciembre de 2012

* Este trabajo muestra resultados parciales del proyecto "Proyección de acciones de política social específicas e inespecíficas en la potenciación de ciudadanía juvenil. Estudio de caso con jóvenes cordobeses de sectores populares", en el Programa "Proyectos de investigación orientados Ciencias Sociales", evaluado y financiado por el Ministerio de Ciencia y Tecnología de la Provincia de Córdoba, Argentina. Instituciones cooperantes: Facultad de Psicología de la Universidad Nacional de Córdoba y el Instituto Académico Pedagógico de la Universidad Nacional de Villa María. Dirección: Dra. Andrea Bonvillani. Una versión preliminar del trabajo fue presentada como ponencia en el XXVIII Congreso Internacional de la Asociación Latinoamericana de Sociología (ALAS) septiembre de 2011. RecifeBrasil, en el Grupo de Trabajo 22 - Sociología de la infancia y la juventud.

* Doctora en Psicología, por la Universidad Nacional de Córdoba. Profesora e investigadora en la Facultad de Psicología de la Universidad Nacional de Córdoba (Argentina). Integrante del equipo de investigación Juventud y nuevas prácticas políticas, Programa de Grupos de Trabajo del Consejo Latinoamericano de Ciencias Sociales (CLACSO). abonvillani@gmail.com

Para

citar este artículo:
Bonvillani, Andrea (2013). “Jóvenes con más y mejor trabajo": desarrollo de sociabilidad grupal juvenil como efecto (inesperado) en la implementación de política pública destinada a jóvenes en la Argentina. Ánfora 20 (34), 15-36. Universidad Autónoma de Manizales. ISSN 0121-6538. 


\section{Resumen}

Objetivos: se indaga sobre los modos como el Estado argentino concibe la cuestión juvenil a través de acciones de política pública que pone en marcha, en especial las que intentan responder a la compleja problemática laboral de jóvenes de sectores populares. Metodología: en el marco de una estrategia de investigación cualitativa, se realizó un análisis documental del Programa sociolaboral del alcance nacional "Jóvenes con Más y Mejor Trabajo" y un ejercicio de reflexión a partir del análisis de los registros de observación participante de los talleres de Educación popular por los que se implementa el Programa en cuestión. Resultados: de la "letra" del Programa, se desprende que éste contempla un conjunto de líneas de acción que superan la mera capacitación laboral, para proyectarse a otras dimensiones - como el ejercicio ciudadano- que se reconocen como intervinientes en el problema del desempleo juvenil, lo cual implica un avance frente a modalidades de intervención estatal propias de décadas pasadas. Del análisis del caso, se destaca la relevancia de la dinámica grupal entre los jóvenes destinatarios (conflictos, alianzas, etc.), en tanto promueve o limita el despliegue de potencialidades juveniles vinculadas al logro del empleo. Conclusiones: el impacto positivo de esta política de empleo no se vincula directamente con el logro de la inserción laboral, sino con el mejoramiento de determinadas condiciones psicosociales favorables a este logro, tales como el afianzamiento de la red grupal, la promoción del protagonismo juvenil y el logro de la terminabilidad educativa.

Palabras claves: inserción laboral, jóvenes, capacitación laboral, política pública.

\section{Abstract}

Objective: to find the view of the Argentinian State regarding the youth issue on the matter of public policy actions it launches, especially those that seek to address the complex employment problem of youth from the public sectors. Methodology: a documentary analysis of the national program "Youth with More and Better Jobs" was conducted. Likewise, a reflection exercise from the observation records analysis of the participants in the popular education workshops, for which the program is implemented, was done. Results: the program "Youth with More and Better Jobs" provides a set of action lines that exceed mere

16 job training to project to other dimensions, such as the exercise of citizenship, 
which are recognized as participants in the youth unemployment problem. This implies an advance with reference to state intervention modalities typical of past decades. The importance of group dynamics among young recipients (conflicts, alliances, etc.) stands out in the case analysis, while promoting or limiting the deployment of youth potential linked to the employment achievement. Conclusions: the positive impact of this employment policy is not directly linked to the achievement of employability, but rather with the improvement of certain psychological conditions, such as network group strengthening, youth leadership promotion and education terminability achievement.

Keywords: employability, youth, job training, public policy.

\section{Resumo}

Objetivo: indagar sobre os modos como o Estado argentino concebe a questão juvenil, segundo as ações de políticas publica que põe em marcha, em especial as que tentam responder à complexa problemática laboral de jovens de setores populares. Metodologia: realizou se um analise documental do programa nacional "Jovens com Mais e Melhor Trabalho" e um exercício de reflexão a partir do analise dos registros de observação participante dos trabalhos práticos de Educação popular, pelos que se realiza este programa. Resultados: o programa "Jovens com Mais e Melhor Trabalho" contempla um conjunto de líneas de ação que superam a mera capacitação laboral, para projetar se a outras dimensões - como exercício cidadão-que se reconhece como intervenientes no problema de desemprego juvenil, o que implica um avance frente a modalidades de intervenção estatal próprias de décadas passadas. Do analise do caso, destaca se a relevância da dinâmica grupal entre os jovens destinatários (conflitos, alianças, etc.), em tanto promove ou limita o desdobramento de potencialidades juvenis vinculadas ao logro do emprego. Conclusões: o impacto positivo desta política de emprego não se vincula diretamente com o logro da inserção laboral, o que quer é o melhoramento de determinadas condições psicossociais favoráveis a este logro, tais como o fiançamento da rede grupal, a promoção do protagonismo juvenil e o logro da terminabilidade educativa.

Palavras chaves: Inserção laboral, jovens, capacitação laboral, política publica. 


\section{Introducción}

Este artículo presenta resultados parciales de una investigación cuyo interrogante central es: ¿de qué manera el Estado argentino concibe la cuestión juvenil a través de las distintas acciones de política pública que pone en marcha, en especial cuando se trata de jóvenes ${ }^{1}$ de sectores populares? Esto implica que la definición y la ejecución de la política social supone la práctica de las miradas estatales respecto a un problema social y de sus afectados, y las formas en que dialécticamente, en el caso de los jóvenes populares en esta investigación, significan su relación con lo estatal mediante sus experiencias cotidianas con dicha política. Según este planteo general del objeto de indagación, se recortan las siguientes preguntas: ¿Cuáles son las concepciones de juventud subyacentes a las políticas sociales destinadas a los jóvenes? ¿De qué modo se conceptualizan y se intenta resolver lo que se considera "problemática juvenil" en estas intervenciones estatales particulares? En consecuencia, este artículo presenta una reflexión crítica que focaliza en los supuestos según los cuales se definen los problemas laborales que, según la mirada estatal, aquejan a los jóvenes de sectores populares y a los alcances de las políticas sociales destinadas a responder a la compleja cuestión de la exclusión social de estos jóvenes.

Se entenderá por "políticas sociales" a las "específicas intervenciones sociales del Estado que se orientan (en el sentido de que producen y moldean) directamente a las condiciones de vida y de reproducción de la vida de distintos sectores y grupos sociales, y que lo hacen operando especialmente en el momento de la distribución secundaria del ingreso" (Danani, 2004, pág. 11). En consecuencia, las políticas sociales operan de manera redistributiva, es decir, no lo hacen regulando ingresos del capital y el trabajo, como las laborales, o distribuyendo ingresos de la producción de manera inmediata (Danani, 2004). Tratándose de sociedades de clases, las condiciones de vida que las políticas sociales expresan y construyen son diferenciadas para cada grupo social. En esta forma, se constituyen en un campo de lucha, puesto que implican el reconocimiento de necesidades y los modos legítimos de su satisfacción. Definida de este modo, la política social constituye "un momento inmediatamente político del proceso de distribución y, por lo tanto, de acumulación” (Danani; citado por Logiudice, 2011, p. 62).

La no diferenciación en el presente escrito entre "las" y "los" jóvenes no debe asociarse a la asunción de una posición discriminatoria en relación a la cuestión del género. Sólo se asume "los jóvenes" en masculino para hacer más fácil la lectura del artículo. 
En este trabajo particular, se analiza el programa "Jóvenes con más y mejor trabajo" que depende del Ministerio de Trabajo, Empleo y Seguridad Social de la Argentina (en adelante, MTEySS) (Ministerio de Trabajo, Empleo y Seguridad social, 2004) en lo referido al modo como se lo presenta en la letra de la resolución que lo reglamenta, y en la reflexión de una experiencia puntual en la que la autora de este artículo participó como coordinadora de uno de los grupos de jóvenes destinatarios de dicha acción.

De este análisis, se desprende como una categoría emergente central la "sociabilidad grupal juvenil"", entendiéndola de manera general como "un modo de estar con el otro" (Duschatzki y Corea, 2005). Para abordarla, es necesario considerar distintas vicisitudes del lazo social: acuerdos, conflictos y formas de resolución que surgen en un devenir grupal y que suponen el desarrollo de vínculos entre los jóvenes. En la medida en que el grupo es el locus en el cual la sociabilidad habrá de desplegarse en interacciones cara a cara, ésta se encuentra articulada con la grupalidad:

Posibilidad, potencialidad de ser grupo. Dimensión con sentido de temporalidad, de proceso, de camino o trayectoria que puede llegar a niveles y grados muy diversos. Hay una posibilidad de devenir, de construir-se, de auto-organizarse abierta y flexiblemente en esta dimensión de grupalidad (Souto de Asch citado por Bonvillani, 2011).

\section{Antecedentes y marco conceptual}

\section{Políticas sociales para los jóvenes: algunas reflexiones generales}

Algunas investigaciones (CEPAL, 1999) (OIT, 1999) (Salvia y Miranda, 2000) ubican a los jóvenes como el grupo social que más ha recibido el impacto negativo de los procesos de ajuste de las últimas décadas en nuestro continente, impacto que se observa especialmente respecto a la desocupación y precarización laboral, que incide decisivamente en sus posibilidades de inclusión social.

1 De la revisión bibliográfica realizada se observa que, a pesar de ser un concepto utilizado en múltiples investigaciones, en relación con la sociabilidad, no abundan las precisiones conceptuales. Un referente clásico en estos temas es George Simmel, para quien las diversas expresiones de sociabilidad remitían a una instancia antropológica universal y permanente, una suerte de constante transhistórica que se manifestaría a través de una constelación de contenidos diferentes que preexisten, sin embargo, a éstos. Se trata de un a-priori socializador que posibilita la existencia de lo societal. En este artículo, se considera que la sociabilidad no es una necesidad humana instintiva universal que a-priori movilizaría a todos por igual, sino, más bien, un proceso psicosocial condicionado por las experiencias particulares de los sujetos que la protagonizan, inscritos en condiciones socio-históricas y culturales que delinean las formas que la sociabilidad va adquiriendo en este devenir colectivo. 
La exclusión social, que afecta a amplios sectores de la sociedad argentina, adquiere en los jóvenes ribetes especialmente preocupantes. En un análisis general del contexto socio-económico argentino post crisis de 2001, se puede admitir un ciclo de crecimiento y una disminución de la proporción de la población en situación de pobreza (Miranda, 2008). No obstante, esta mejora no parece traducirse en la situación juvenil. En la Encuesta permanente de Hogares de 2005 (Salvia et al., 2008) estiman que en estratos bajos el $20 \%$ de los jóvenes de 15 a 19 años no estudia ni trabaja, porcentaje que crece a casi el 37\% cuando se trata de jóvenes entre 20 a 24 años.

Muchos autores han señalado que la centralidad del trabajo en la estructuración de la sociedad moderna, como eje articulador de múltiples proyectos, dio forma a la experiencia cotidiana de los sujetos en los registros socioeconómico, político, educativo y en acciones de política pública. Hoy se sabe que gran parte de las transformaciones socio-culturales ocurridas recientemente han corrido de ese centro al trabajo y, en consecuencia, someten a las personas a una intensa búsqueda de reestructuración de su propia existencia, con el telón de fondo de la desafiliación (Castel, 2004) cuando se trata de sectores empobrecidos. Este proceso ha afectado especialmente a los jóvenes, en la medida en que la inserción en el medio laboral significó hasta hace poco, su ingreso a la vida adulta, por lo menos en los cánones más estabilizados de la sociedad moderna:

Al perder capacidad real y simbólica el concepto de trabajo tradicional y sus instituciones derivadas, encargadas de socializar e integrar a las nuevas generaciones con base en el estatuto salarial y como eje organizador de la sociedad (...), su proceso de incorporación social, se ha ido moviendo a una diferente manera de afrontar la situación y lograr, si no la integración, cuando menos la sobrevivencia ante las nuevas situaciones (Pérez-Islas, 2008, p. 178).

En la década del noventa, se hizo especialmente evidente que las transiciones que tradicionalmente aseguraban el paso de los jóvenes a la vida adulta estaban muy lejos de ser lineales, sobre todo porque las transformaciones en el sistema productivo y la crisis de las instituciones sociales (familia, escuela) afectaron severamente el desarrollo de la autonomía juvenil. En nuestra región, los fuertes cambios ocurridos al calor del neoliberalismo en esa década (sobre todo la flexibilización laboral) y el aumento concomitante del desempleo y la pobreza, tuvieron a los jóvenes como sus blancos privilegiados. Algunos de los rasgos definitorios de la relación de los jóvenes de sectores populares con el trabajo son, desde aquella época, la informalidad, la intermitencia, la devaluación de credenciales, y especialmente el desempleo y la precariedad (OIT, 2004) (CEPAL, 2007) (Jacinto, 2008). 
Consecuentemente, la ecuación juventud-pobreza fue incorporada a la agenda de las políticas sociales como uno de sus temas principales:

Esta población (...) pasó a constituirse en un segmento vulnerable sobre el cual el Estado debía emprender acciones de capacitación profesional y participación comunitaria, con el fin de facilitar su inclusión competitiva en un mercado cada vez más exigente, lo cual se consideraba condición necesaria para salir de la pobreza (Salvia, de Souza, Schmid, Scofienza, y van Raap, 2006, p. 5).

No obstante, en concordancia con una imagen de juventud como problema (Chávez, 2005), siempre presente en el imaginario social, este tipo de medidas se consideraron necesarias en el horizonte del control del conflicto social. En esta forma, se actualizó para los jóvenes pobres una premisa que suele atribuirse a la política social, que es la de "normalizar" a los sectores más postergados, que son visualizados como peligrosos para la propiedad y el orden social (Andrenacci y Soldano, 2005). En la década del noventa, las intervenciones estatales dirigidas a favorecer las condiciones de inserción educativa y ocupacional de los jóvenes estuvieron animadas por dos líneas diagnósticas en torno al problema del desempleo juvenil (Salvia, Schmid, Scofienza, y van Raap, 2006): la existencia de "barreras internas", es decir, calificaciones insuficientes, falta de experiencia, etc. O "barreras externas", esto es, regulaciones del mercado laboral que se consideraban "rígidas", transformaciones tecnológicas del sistema productivo. Así lo muestran las acciones desplegadas por el Estado en ese momento para atender las cuestiones de desempleo y retraso educativo de los jóvenes, las que siguiendo a Salvia y Tuñón (2005), son básicamente tres:

- Reformulación de sistemas de formación técnico profesional, educación media y superior y ampliación de años de escolaridad obligatoria, en aplicación de la Ley Federal de educación y Educación superior promulgada en Argentina en 1993;

- Promoción de modalidades flexibles de contratación laboral para favorecer la demanda juvenil;

- Asistencia económica con el objetivo de lograr la permanencia en el nivel escolar secundario o la formación técnica para el trabajo, por medio de becas escolares para adolescentes de familias pobres y programas de capacitación para jóvenes con déficit educativo en igual situación.

Los Programas que se implementaron en esta época, relativos a sectores juveniles de escasa calificación, se concentraron en la capacitación laboral según el supuesto de que el déficit de empleabilidad juvenil era consecuencia de su 
escaso capital humano ${ }^{1}$ (Jacinto, 2008). Estas acciones estuvieron orientadas al mercado laboral formal e incluyeron pasantías en empresas, pero en la práctica los certificados otorgados no lograron ningún tipo de reconocimiento en la formación profesional regular, sobre todo porque la mayoría de los jóvenes destinatarios no contaba con el título de nivel secundario. De este modo, los programas se encontraban desarticulados de la educación formal. Además, retomando los signos propios de la matriz asistencial neoliberal, estos programas eran muchas veces efímeros y discontinuos, pues estaban subordinados a la discrecionalidad gubernamental. Así, se puede concluir con Jacinto (2008) que:

La mayoría de las iniciativas mostraron cuando menos una simplificación de la problemática de la inserción de los jóvenes en el mercado de trabajo, en particular la de los jóvenes de sectores más pobres. Las condiciones estructurales más duras que mostraban tanto un deterioro general del mercado de trabajo como la configuración de modelos societales excluyentes, la debilidad de las instituciones educativas, y las condiciones subjetivas de los propios jóvenes, sus relaciones y estrategias en relación al trabajo, no ocuparon lugares centrales en los diagnósticos ni en las acciones" (p. 127).

Estas marcas fundacionales según las cuales se acuñaron las políticas sociales destinadas a los jóvenes en los noventa persisten hasta hoy, dado que "la definición de la problemática juvenil continúa centrada en los jóvenes de familias pobres o marginales que no estudian ni trabajan, como consecuencia de sus déficit de capital humano" (Salvia et al., 2006, p. 7).

Estos análisis indican que se continúa eludiendo la inscripción de la cuestión en causas estructurales o institucionales profundas, lo que impide ubicar la falta de empleo como un síntoma de la propia desafiliación (Salvia et al., 2006). Las condiciones de inserción laboral para los jóvenes pobres son aún especialmente desfavorables frente a otros segmentos poblacionales. La segregación residencial, la segmentación social y la devaluación de credenciales, siguen delineando las desventajas de todo tipo para estos jóvenes. Una característica de la estructura del mercado laboral de los últimos años parece ser la acentuación de la baja calidad de los empleos, lo cual se traduce en la obtención de puestos con alta rotación, temporales o eventuales.

Jacinto (2008) ha reseñado algunos cambios que se han planteado en relación con las estrategias de intervención estatal frente al problema de la desocupación juvenil:

La empleabilidad es definida como la aptitud para "encontrar, crear, conservar enriquecer un trabajo y pasar de uno a otro obteniendo a cambio una satisfacción personal, económica, social y profesional" (MTEySS, 2004, p. 9). 
a. Énfasis en la terminación de la escolaridad secundaria, a partir de lo cual se privilegian la retención y la re-inserción en el sistema educativo formal. Esto indica una concepción de la empleabilidad vinculada con la titulación a través de las instituciones formales y, además, la puesta en valor de la "adquisición de competencias transversales tanto para la vida ciudadana como para la laboral" (Jacinto, 2008, p. 134).

b. Dentro de un clima de época marcado por la progresiva pérdida de densidad de la cultura del trabajo como eje articulador de la identidad social juvenil, se observa una suerte de cambio actitudinal frente al trabajo, es decir, una escasa motivación para tal práctica de los jóvenes: "De este modo, se comienza a configurar un cambio en los ejes conceptuales a partir de los cuales se abordan los problemas de la inserción laboral de los jóvenes: de las "necesidades de capacitación" hacia las "disposiciones hacia el empleo" (Jacinto, 2008, p. $130)^{1}$. Este viraje se pone en evidencia en la tendencia a superar la capacitación técnica como único propósito, para incluir componentes que apuntan a otras dimensiones del problema, tales como orientación y acompañamiento en el proceso de inserción laboral. Estos componentes apuntan a incidir sobre aspectos subjetivos de los destinatarios, en el supuesto de que se dan actitudes o disposiciones en ellos que dificultan su inserción laboral, tales como autoestima, motivaciones, expectativas, etc. y que, consecuentemente, su reforzamiento contribuiría al aumento de empleabilidad. Por lo general, las acciones que vehiculizan estos objetivos tienen que ver con el acompañamiento a los jóvenes en la evaluación de su propio perfil, las estrategias en la búsqueda de empleo, la manera de enfrentar una entrevista laboral, etc., hasta metodologías más complejas basadas en pedagogías activas, tales como los denominados "proyectos ocupacionales" (Silveira; citado por Jacinto, 2008) En esta misma línea, las pasantías o programas de primer empleo suponen experiencias de socialización laboral, porque le permiten al joven la experiencia de vivir bajo las reglas del mundo del trabajo, y se constituyen en un "puente con el empleo" (Jacinto y Millenaar, 2009). No obstante, entre sus aspectos negativos, se apunta la escasez de lugares concretos donde realizarlas y los abusos patronales cuando se implementan como formas encubiertas de empleos de bajísima remuneración.

Planteamiento diagnóstico cercano a una nueva forma de hacer recaer la responsabilidad del desempleo a los propios jóvenes, esta vez con el riesgo agregado de caer en la sanción moral de la "vagancia" según la cual se suele juzgar a los sectores poblacionales de pobreza en general. 
c. En algunos Programas, aparece aludida la cuestión de la "inclusión ciudadana”, como uno de los propósitos de la intervención. En este punto conviene prestar atención a los enfoques conceptuales desde los cuales se parte:

Mientras que algunas se apoyan en una concepción del joven como problema y se insertan en iniciativas más amplias vinculadas a la seguridad ciudadana, otras se ubican desde la promoción de "los derechos de los jóvenes", intentando abordajes multisectoriales y el protagonismo de los propios jóvenes" (Jacinto, 2008, p. 131).

\section{Metodología}

La investigación es de orientación cualitativa, en tanto se propone identificar y describir las concepciones sobre la cuestión juvenil que subyacen al diseño y la implementación de políticas sociales para jóvenes en la Argentina contemporánea, además de producir una reflexión crítica sobre el impacto cualitativo en una experiencia puntual de aplicación. De este modo, en una primera etapa, se realizó un relevamiento y el análisis documental de programas y proyectos estatales dirigidos a la juventud en el país, lo que permitió la construcción de un mapa de éstos que, por su extensión, excede el marco de este artículo. En un segundo momento, se seleccionó para un análisis focalizado el programa sociolaboral de alcance nacional "Jóvenes con más y mejor trabajo", para lo cual se tomó, como documento base, la resolución ministerial que lo reglamenta. Esta Resolución fue sometida a "análisis documental" (Vallés, 1999). Finalmente, se realizó un ejercicio de reflexión a partir del análisis de los registros de la observación participante de los talleres de educación popular que la autora de este artículo coordinó. Estos talleres constituyeron la instancia de implementación del programa en una localidad del interior de Argentina. Se siguieron los lineamientos de la observación, registro y análisis propios de la Etnografía (Guber, 2011). Dicho análisis permitió poner en tensión la "letra" del programa y su momento de implementación en los talleres, a los fines de observar las distancias entre concepciones y marcos ideológicos que se asumen conceptualmente en la política social y las prácticas que ésta implica.

A continuación, se ofrece un análisis de los artículos más importantes de la Resolución 497/2008 del MTEySS, que reglamenta los componentes y las acciones que integran el Programa "Jóvenes con más y mejor trabajo", y, luego, mostrar de qué modo lo expresado en la letra, se concreta en una experiencia de

24 aplicación concreta, teniendo en cuenta las siguientes ejes analíticos: 
- Propósitos y objetivos del Programa;

- Prestaciones declaradas del Programa y su aplicación en los talleres grupales en los que participaron los jóvenes destinatarios;

- Concepciones del desempleo juvenil en las líneas de acción que el Programa enuncia y su relación con los impactos y resultados inmediatos de su implementación en una experiencia concreta.

\section{Resultados}

Programa "Jóvenes con más y mejor trabajo": análisis del marco normativo

El Programa "Jóvenes con más y mejor trabajo" fue reglamentado a través de una resolución del MTEySS, sancionada en mayo de 2008. Su propósito fundamental es:

Generar oportunidades de inclusión social y laboral de las y los jóvenes a través de acciones integradas que les permitan identificar el perfil profesional en el cual deseen desempeñarse, finalizar su escolaridad obligatoria, realizar experiencias de formación y/o de prácticas calificantes en ambientes de trabajo, iniciar una actividad productiva de manera independiente o insertarse en un empleo (Artículo primero, Resolución 497/2008, MTEySS).

En el artículo tercero, se destaca que las prestaciones del programa servirán de apoyo a la construcción y la implementación del proyecto formativo y ocupacional que cada joven decida, con el objetivo de proporcionarles oportunidades de desarrollar trayectorias laborales pertinentes y de calidad, adecuadas a sus perfiles, a sus expectativas y a sus entornos. De este modo, el programa articula varios ejes de prestación:

a. Orientación e inducción al mundo del trabajo. Durante el desarrollo de este proceso, se fortalecerá al joven participante en la actualización, revisión o construcción de su proyecto formativo y ocupacional. Para ello, se le brindarán elementos para la identificación de: i) sus intereses, necesidades y prioridades y su vinculación con un proyecto formativo y ocupacional; ii) las particularidades de su entorno social y productivo para elegir estrategias de formación y trabajo; iii) los saberes y habilidades para el trabajo que haya adquirido en distintos espacios de aprendizaje y experiencia; y iv) estrategias adecuadas para planificar y desarrollar su itinerario de formación, búsqueda y acceso al empleo. Esta etapa es obligatoria y previa a las demás y sólo podrán desarrollarse en forma simultánea a ella los estudios primarios y secundarios. 
b. Formación para la certificación de estudios primarios y secundarios. Esta prestación es una prioridad estratégica para la inclusión ciudadana y, desde el punto de vista de la inclusión laboral, es una de las vías para mejorar la inserción de los jóvenes en trabajos de calidad y disminuir la rotación exacerbada en empleos de corta duración.

c. Cursos de formación profesional. De acuerdo con sus intereses y expectativas de inserción laboral, los jóvenes podrán participar en cursos de formación profesional que les permitirán adquirir o fortalecer las competencias y habilidades requeridas para el ejercicio de la ocupación definida durante la etapa de elaboración de su proyecto formativo y ocupacional.

d. Certificación de competencias laborales. Los jóvenes que han tenido experiencia laboral previa podrán ser evaluados y certificadas las competencias laborales que han desarrollado en el ejercicio de esa ocupación.

e. Generación de emprendimientos independientes. Los jóvenes que opten por desarrollar su propio emprendimiento, de manera individual o asociativa, serán apoyados a través de asistencia técnica y de cursos de capacitación en la formulación de su plan de negocio.

f. Prácticas calificantes en ambientes de trabajo, tendientes a iniciar o completar la formación recibida en empresas del sector público o privado, para lo cual recibirán asesoramiento de las oficinas de empleo municipales.

g. Apoyo a la búsqueda de empleo. Los jóvenes recibirán de manera permanente asistencia para la elaboración de estrategias adecuadas para la búsqueda de empleo.

h. Intermediación laboral. A través de las oficinas de empleo municipales, los jóvenes incorporados al programa serán informados sobre las demandas de trabajo formuladas por las empresas que sean compatibles con sus perfiles profesionales.

i. Apoyo a la inserción laboral. Se promoverá la contratación de jóvenes, ofreciendo incentivos financieros, por un plazo máximo de seis meses, a las micro, pequeñas y medianas empresas que los incorporen como trabajadores en relación de dependencia.

Como se desprende de la letra del Programa, éste contempla un conjunto de líneas de acción que superan la mera capacitación laboral, para proyectar26 se a otras dimensiones que se reconocen como intervinientes en el problema 
del desempleo juvenil. Evidentemente, esto implica una mirada integral sobre la cuestión, si la comparamos con aquella que vinculaba el desempleo juvenil con una "inadecuación" de las competencias juveniles frente a la demanda del mercado laboral, sobre todo porque se parte de la elección y la elaboración de un proyecto formativo y ocupacional del cual el propio joven es agente, regido por sus propios "intereses y expectativas de inserción laboral", lo cual se asume como criterio rector de todas las prestaciones del Programa.

De este modo, los distintos componentes que articulan el Programa parecen mostrar una sensibilidad respecto de la problemática del desempleo juvenil conceptualizada como una "constelación de desventajas" (Jacinto y Millenaar, 2009), para aludir a la compleja articulación entre el acceso a oportunidades y recursos (dimensión socio-económica e institucional) y las formas de acuerdo que los propios sujetos perciben, dan sentido y gestionan esos recursos. Por ello, la consideración de la dimensión subjetiva respecto de las cuestiones de desempleo, fundamenta la importancia que se le otorga al módulo orientación e inducción al mundo del trabajo, en el cual se reflexiona sobre expectativas y actitudes frente a la cuestión, así como se recuperan saberes de los jóvenes adquiridos en sus propias experiencias previas.

Otro elemento que debe destacarse es la centralidad de la terminalidad educativa dentro del Programa, subrayándola no sólo por su vinculación con la inclusión laboral, sino también con el ejercicio de la ciudadanía. De esta forma, vemos actualizarse lo que señala Jacinto (2008) como uno de los rasgos que caracterizan las acciones actuales destinadas a lograr incrementar la empleabilidad juvenil, pero al mismo tiempo incorporar competencias para la vida ciudadana. En este sentido, también puede interpretarse el hecho que el Programa incluya dos módulos (derechos laborales y sociales y condiciones de trabajo y salud ocupacional) en los que se concientiza a los jóvenes sobre sus derechos como trabajadores, y las vías para reclamar su vigencia.

\section{Programa "Jóvenes con más y mejor trabajo": apuntes sobre una experiencia de implementación}

En la provincia de Córdoba (Argentina), se desarrolló este Programa durante 2009 y 2010, como experiencia piloto en la ciudad de Villa Allende, cercana a la capital de la provincia. Las entidades coordinadoras locales fueron la Secretaría de Extensión de la Universidad Nacional de Córdoba y la Oficina de Empleo de la Municipalidad de Villa Allende. A continuación, se presentan algunas reflexiones en torno al proceso de trabajo realizado bajo mi coordinación técnica, con un grupo 
que osciló entre veinte jóvenes al principio y quedó constituido por doce. El trabajo se desarrolló durante trece encuentros en los meses de junio y julio de 2009, en desarrollo de dicho programa. La modalidad de trabajo fue el taller. A través de distintas técnicas (recreativas, expresivas, cognitivas) se desplegaron los objetivos correspondientes al módulo "Construcción de un proyecto formativo y ocupacional"1.

El grupo de jóvenes presentaba las siguientes características:

- En su mayoría, estaba compuesto por jóvenes mujeres con hijos, quienes, en el momento de los talleres, estaban sin pareja y convivían con sus padres o con otros familiares.

- Procedían de barrios populares, desprovistos de condiciones de infraestructura como pavimento en sus calles. Los propios jóvenes retomaban para su caracterización los juicios sociales negativos según los cuales se los ve como pobres e inseguros.

- La mayor parte del grupo no había terminado el nivel medio, mientras que dos jóvenes tenían el primario incompleto.

- La ocupación que habían desarrollado con más frecuencia los varones era la albañilería, y las mujeres tareas domésticas y de comercio. Este dato está en consonancia con la caracterización del contexto laboral de la ciudad de Villa Allende, puesto que, al desagregar las distintas ramas de actividad según la cantidad de ocupados de dicha localidad ${ }^{2}$, se puede observar que los sectores de servicios son los mayores demandantes de mano de obra. Por otra parte, se pueden considerar las bajas calificaciones requeridas a los trabajadores según las ramas predominantes de actividad. Los jóvenes referían frecuentes dificultades para conseguir empleo y mantenerlo en el tiempo, en su breve experiencia personal y respecto a la trayectoria que sus familiares cercanos (padres, hermanos, tíos) habían desarrollado. Las referencias en este punto indican una cadena de precariedad e inestabilidad laboral marcadas.

1 Las temáticas desarrolladas en este espacio fueron: relato de la historia personal y de la trayectoria educativa-laboral de los jóvenes, identificación de recursos personales (competencias y habilidades), y sus limitaciones relativas, deseos y expectativas en relación con su inserción laboral, problematización de la distribución sexual del trabajo, identificación de demandas, oportunidades y requerimientos del contexto laboral nacional y regional, desempleo juvenil, herramientas básicas para la confección del propio curriculum vitae, etc. Otros componentes del Programa fueron: informática básica, derechos laborales y sociales y condiciones de trabajo y salud ocupacional.

2 Instituto Nacional de Estadísticas y censos (INDEC)- Encuesta Permanente de Hogares $1^{\circ}$ Trimestre 2007. juvenil como efecto (inesperado) en la implementación de política pública destinada a jóvenes en la Argentina. Ánfora 20 (34), 15-36. Universidad Autónoma de Manizales. ISSN 0121-6538. 
El micro-proceso que el grupo desarrolló puede reseñarse a partir de la reconstrucción de tres momentos:

- La apertura, en los primeros encuentros. En este momento lideró el proceso un sub-grupo constituido por cuatro jóvenes que se conocían con anterioridad (algunos eran parientes) y que tendían a hegemonizar la palabra y a centralizar las interacciones con la coordinadora. Debido a su estilo comunicacional un tanto agresivo, de algún modo inhibían a los demás miembros del grupo, lo que dificultaba el desarrollo de la grupalidad. Incluso, una de las jóvenes plantea en el primer encuentro no querer trabajar con una de ellas, debido a que se "llevan muy mal", con lo que actualizaba conflictos no resueltos de instancias barriales. Al principio, fue difícil lograr que abordaran las consignas puesto que debían "exponerse" frente al grupo para leer su producción en el taller. Esta dificultad remite a experiencias educativas anteriores en las cuales se sintieron descalificados, tal vez por no ajustarse a los requerimientos de esos espacios. Además, algunos de ellos, aún reconociendo que sabían leer, mostraban en el momento de la socialización de lo producido, algunas dificultades para la lectura con fluidez.

- Luego, dicho sub-grupo comienza a faltar reiteradamente por problemas de salud de familiares, y sólo uno de sus miembros, la joven que muestra más resistencias a las pautas del encuadre, continúa asistiendo. Se produce una suerte de reacomodamiento grupal: ahora ella se integra con el resto del grupo y trabaja sin mayores dificultades. Por su parte, la disolución temporaria del sub-grupo imprime una nueva dinámica al colectivo: las interacciones se hacen más fluidas y comienzan a establecerse lazos de afinidad entre algunos miembros que se han conocido en los talleres, sobre todo entre aquellos que asistieron desde el principio. Se incorporan nuevos jóvenes, mientras que otros dejan de asistir. La inestabilidad en la conformación del grupo dificulta el proceso de trabajo, puesto que implica explicitar el encuadre una y otra vez, lo cual resta tiempo y cansa a los que vienen desde un principio. Además, afecta la consolidación de lazos grupales que faciliten el desarrollo del proceso grupal. Una explicación para las dificultades de retención de los jóvenes es la demanda de tiempo que exige el programa (seis horas por día, de lunes a viernes), lo que pudo haber causado una suerte de rutinización para cumplir con la asistencia, y afectar el aprovechamiento efectivo de estos espacios.

- Hacia el cierre, hubo jóvenes que no se integraron al colectivo y aparecieron como "aislados" frente a los dos sub-grupos que se fueron constituyendo: el 
que apareció al inicio -que en realidad era previo a la propia experiencia- y el que se fue consolidando a instancias de la implementación del Programa. Este último se constituye en uno de los "productos psicosociales" destacados del proceso vivido por los jóvenes: ellos mismos reconocieron que lo que se "llevaron" del taller fue el conocerse y, a partir de esta experiencia, contar los unos con los otros cuando ya no fueran más, por ejemplo, en el momento que tuvieran que retomar la escuela.

Este breve análisis, permite mostrar cómo la sociabilidad grupal (conflictos, alianzas) es una dimensión relevante en la puesta en práctica del programa, pues opera promoviendo o limitando el despliegue de potencialidades juveniles. Por ejemplo, cuando se dan tensiones irresolubles entre los participantes, la producción colectiva se resiente, puesto que hace improbable el establecimiento de acuerdos mínimos de trabajo, lo que, debido al escaso tiempo frente a la cantidad de temas que se deben abordar en los talleres, no encuentra oportunidad de ser elaborado colectivamente como parte de las intervenciones del coordinador. Esto creó un clima de tensión en la dinámica grupal que, por ejemplo, pudo inhibir el despliegue de la producción personal frente al grupo y, quizás, limitó las posibilidades de completar ciertos aprendizajes que hacen al desarrollo de autopercepciones positivas en los jóvenes.

Con estas reflexiones no se propone evitar el conflicto como estrategia de trabajo, pues resulta evidente que en la construcción grupal este es inherente (Bonvillani, 2011). Este apunte se hace para poner en visibilidad la cuestión de la sociabilidad grupal como una de las líneas de acción que este programa debería tomar en consideración, sobre todo porque su implementación supone como recurso didáctico el dispositivo del taller, es decir, la experiencia de construcción colectiva de saberes.

Efectivamente, el grupo opera como soporte psicosocial del programa en varios sentidos. Por un lado, ofrece un escenario de interacción cara a cara con pares y adultos que, en muchos casos, favorece la reparación de los efectos negativos de experiencias escolares anteriores que han sido altamente descalificantes para los jóvenes. El comprobar que la propia palabra es tenida en cuenta y valorada, produce efectos confirmatorios que tienden a instalar capacidades expresivas en los jóvenes, tal vez menguadas en parte por las evaluaciones reprobatorias de las que suelen ser objeto en su paso por la escolaridad obligatoria, según ellos mismos lo han referido a instancias de su participación en los talleres. 
En esta dirección, uno de los logros destacables tiene que ver con el desarrollo de capacidades de expresión oral. Algunos de ellos, que se expresaban tímidamente al principio y casi "por obligación" frente al requerimiento del coordinador, al final del proceso, lo hacían con soltura y manifestando placer. Uno de los jóvenes, que no se animaba a escribir en su hoja, al final, terminó escribiendo en el pizarrón las consignas para sus compañeros. Otro pudo reconocer frente al grupo que no "sabía leer muy bien, pero que quería aprender a hacerlo". Además de las consideraciones evidentes respecto de la positividad de estos cambios en el plano subjetivo -frente a trayectorias atravesadas por procesos de estigmatización social y de falta de reconocimiento de todo tipo-, éste es un elemento destacable, como competencia psicosocial preparatoria para conseguir empleo y, sobre todo, para luchar por la vigencia y expansión de sus derechos ciudadanos. La sociabilidad grupal que se desarrolla entre los jóvenes, a propósito del encuentro en los talleres, se constituye en capital social en tanto consideran los vínculos interpersonales que allí se van tramando como recursos disponibles, que pueden ser actualizados ante diversas necesidades, como por ejemplo apoyarse en la re-inserción en la escuela.

Si bien muchos estudios (Jacinto y Millenaar, 2009) ponen en evidencia que las redes de relaciones sociales inciden significativamente en las oportunidades de inserción laboral de los jóvenes, lo que en este caso se quiere poner en visibilidad es que como un efecto no previsto del programa, se produce a instancias de su implementación, el establecimiento y afianzamiento de afianzan los lazos de solidaridad entre los jóvenes que se encuentran en situaciones análogas, lo cual constituye un recurso para sostenerse mutuamente en la búsqueda del empleo, por ejemplo. Esta apelación al desarrollo de lazos sociales entre los jóvenes, a manera de apoyo y contención mutua como recurso potencial de empleabilidad, no debe inscribirse en cierta concepción de "privatización de la política social" (Bustelo, 2003, p. 137) que desliga al Estado de sus responsabilidades como garante del bienestar social, y hace recaer la salida a las desigualdades sociales en la solidaridad entre pobres. Sin embargo, es posible identificar como un efecto quizá inesperado pero positivo en la implementación de política pública destinada a originar de manera indirecta condiciones favorables al empleo juvenil.

\section{Conclusiones}

¿Por qué interrogar las formas como son construidas discursivamente las problemáticas sociales juveniles según las políticas sociales destinadas a este sec- 
tor poblacional? Se ha sostenido aquí que las acciones de política social expresan los modos prevalecientes a partir de los cuales el Estado concibe a los destinatarios de dichas acciones y su universo de necesidades y potencialidades. A la vez, es evidente que estas acciones indican el marco interpretativo y los modos de abordaje político-estatal de la relación social, en la medida en que "la política social implica también la distribución material de lo que se acumula socialmente; por esto se instala en un ámbito de disputa y conflicto; es decir, es esencialmente política" (Bustelo, 2003, p. 141). Bajo estos supuestos, se interrogaron propósitos y estrategias enunciados en la letra de uno de los programas estatales destinados a la inclusión socio-laboral juvenil de alcance nacional, actualmente vigente: el denominado "Jóvenes con más y mejor trabajo", y se analizaron aspectos de su implementación en una experiencia concreta.

En el aspecto discursivo, resulta evidente que este programa supera con creces los que respondían a la matriz neoliberal de asistencia, sobre todo porque se abandona la idea de "déficit de empleabilidad", lo cual implicaba la atribución individual del desempleo y, con ella, la culpabilización de los desempleados por esta condición. Por el contrario, aquí encontramos una revaloración de las experiencias y competencias de los jóvenes, así como de las propias capacidades y derechos para "diseñar" su proyecto ocupacional, es decir, el supuesto que parece informar estas acciones se acerca a una definición de la problemática laboral de los jóvenes de sectores populares que no los concibe como culpables o deficitarios, sino que intenta "empoderarlos", capitalizando sus trayectorias laborales y educativas previas.

Lo que sucede es que cuando estas formulaciones del programa se ponen en tensión con el escenario de inserción laboral "real” y, más aún, con las condiciones estructurales en las que transcurre la vida cotidiana de los jóvenes, emerge la pregunta: ¿se trata de intervenciones estatales que verdaderamente tienden a la redistribución o el programa se inscribe más bien en un mero ejercicio de "retórica antineoliberal" (Svampa, 2008)? Esta duda surge porque, en la práctica, al concluir la ejecución, se observaron escasos impactos en el logro de un empleo, aunque los resultados se orientaron a la continuación de los estudios interrumpidos.

Por ello, a pesar que el programa se denomina "Jóvenes con más y mejor Trabajo", puede considerarse que, por las condiciones específicas de su implementación, el objetivo central es incentivar a los jóvenes a que se rein- 
serten en el sistema educativo para que puedan acreditar el título de la educación media. Este no parece un objetivo menor, puesto que es conocido que se trata no sólo de aumentar las posibilidades de conseguir empleo, al menos en un mercado laboral en el que aún se valoran las credenciales, y también porque se trata de posibilidades de ampliar la ciudadanía de los jóvenes de sectores populares, en la medida en que los anima a reinsertarse en el "mercado de bienes simbólicos" en una cultura que, aunque hegemónica, podría constituirse en la base mínima en la cual pueden pensar y articular procesos de resistencia de algún tipo.

Evidentemente, las posibilidades de incidir en el desarrollo de competencias expresivas y de asertividad social, y en el afianzamiento de lazos de grupalidad que exceden la propia duración del programa, depende, en parte, de que el dispositivo de implementación las favorezca: "sin duda los abordajes institucionales y pedagógicos, el entramado de actores participantes y la calidad de la oportunidad de aprendizaje y experiencia influyen en los resultados" (Jacinto, 2008, p. 136). La concientización de los jóvenes respecto de sus derechos ciudadanos, para que adquieran herramientas subjetivas en la defensa contra la explotación a que los somete el mercado de trabajo (informalidad, precarización, etc.), tuvo una fuerte impronta al menos en la experiencia de implementación del programa que aquí se reseñó: ellos mismos reconocieron la importancia de "conocer sus derechos para no dejarse explotar".

En síntesis, algunos elementos de incidencia de políticas sociales destinadas a la población juvenil popular derivados de la investigación que aquí se reporta son los siguientes:

- El impacto positivo de esta política de empleo no se vincula directamente con el logro inmediato de la inserción laboral, sino con el mejoramiento de determinadas condiciones psicosociales favorables a este logro, tales como el afianzamiento de la red social y la promoción del protagonismo juvenil, de acuerdo con otros reportes de investigación similares (Farías, 2011).

- En este marco, se recorta como central la promoción de procesos grupales que favorezcan el desarrollo de sociabilidad grupal juvenil en el escenario del taller. Esto supondría desarrollos colectivos de más tiempo e intensidad, que luego podrían ser capitalizados por los jóvenes participantes como lazos sociales de contención y ayuda mutua en la búsqueda de empleo y en el logro de la culminación educativa. En tal sentido, es necesaria la introducción de 
un "dispositivo de intervención grupal" que disponga deliberadamente el trabajo en equipo y sea capaz de analizar los procesos vinculares de los jóvenes, para que no se trate de un producto psicosocial contingente, sino que busque de manera intencional en el programa, para afianzar lazos de grupalidad, solidaridad y compromiso con la acción colectiva.

- Mientras no se modifiquen las condiciones objetivas en las cuales los jóvenes se insertan laboralmente, este tipo de programas resulta un paliativo que continuará poniendo en evidencia un conflicto inherente a la política social, en tanto esta, como "construcción de igualdad, alude esencialmente a la justicia distributiva" (Bustelo, 2003, p. 142), dejando como deuda pendiente las posibilidades de verdaderas trasformaciones en la inserción social para los jóvenes de sectores populares en Argentina a partir de las respuestas estatales.

\section{Referencias}

Andrenacci, L. y Soldano, D. (2005). Aproximación a las teorías de la política social a partir del caso argentino . En L. A. (comp.), Problemas de politica social en la Argentina contemporánea. Buenos Aires: Prometeo.

Bonvillani, A. (2011). Travesías grupales. Algunas coordenadas para pensar/trabajar con grupos. Córdoba: Universidad Nacional de Córdoba.

Bustelo, E. (2003). ¿Retornará lo social? Íconos, Revista de Ciencias sociales 17, 133143.

Castel, R. (2004). La metamorfosis de la cuestión social. Buenos Aires: Paidós.

CEPAL (1999). Panorama Social de América Latina. Santiago de Chile. Santiago de Chille: Comisión Económica para América Latina y el Caribe.

CEPAL (2007). Panorama Social de América Latina 2007. Santiago de Chile: Comisión Económica para América Latina y el Caribe.

Chávez, M. (2005). Juventud negada y negativizada: representaciones y formaciones discursivas vigentes en la Argentina contemporánea. Última Década 23, 9-32.

Danani, C. (2004). El alfiler en la silla: sentidos, proyectos y alternativas en el debate de las políticas sociales y la economía social. En C. Danani, Política social y economía social. Debates fundamentales. Buenos Aires: UNGS-Alt. 
Duschatzki, S., y Corea, C. (2005). Chicos en banda. Buenos Aires: Paidós.

Farías, M. (2011). Reflexiones en torno al impacto de programas de empleo para jóvenes en Argentina. Un estudio de caso. Última Década 35, 169-194.

Guber, R. (2011). La etnografía. Método, campo y reflexividad. Buenos Aires: Siglo XXI .

Jacinto, C. (2008). Los dispositivos recientes de empleo juvenil: institucionalidades, articulaciones con la educación formal y socialización laboral. Revista de Trabajo 6, (4), 123-142.

Jacinto, C., y Millenaar, V. (2009). Enfoques de programas para la inclusión laboral de los jóvenes pobres: lo institucional como soporte subjetivo. Última Década 30, 67-92.

Logiudice, A. (2011). Pobreza y Neoliberalismo: La asistencia social en la Argentina reciente. Entramados y perspectivas. Revista de la Carrera de Sociología de la Universidad Nacional de Buenos Aires 1, 61-90.

Ministerio de Trabajo, Empleo y Seguridad social. (2004). El Proyecto Ocupacional. Una metodología de formación para mejorar la empleabilidad. Buenos Aires: CINTERFOR/OIT.

Miranda, A. (2008). La inserción laboral de los jóvenes en Argentina. En M. H. R. Bendit, Los jóvenes y el futuro. Procesos de inclusión social y patrones de vulnerabilidad en un mundo globalizado. Buenos Aires: Prometeo.

OIT (1999). Informe sobre el empleo en el mundo 1998-1999. Ginebra: Oficina Internacional del Trabajo.

OIT (2004). Tendencias mundiales del empleo juvenil. Ginebra: Organización Internacional del Trabajo.

Pérez Islas, J. A. (2008). Entre la incertidumbre y el riesgo: ser o no ser, esa es la cuestión juvenil. En M. H. R. Bendit, Los jóvenes y el futuro. Procesos de inclusión social y patrones de vulnerabilidad en un mundo globalizado. Buenos Aires: Prometeo.

Salvia, A. d., Schmid, S., Scofienza, M. A. y van Raap, V. (2006). Los jóvenes pobres como objeto de políticas públicas zuna oportunidad para la inclusión social o un derrotero de manipulación y frustraciones? (Ponencia presentada en el Tercer Congreso de Políticas Sociales). Buenos Aires: Observatorio Social de la Universidad Católica de Buenos Aires.

Salvia, A. et al. (2008). Jóvenes promesas. Trabajo, educación y exclusión social de jóvenes pobres en la Argentina. Buenos Aires: Universidad Nacional de Buenos Aires y Miño y Dávila Ediciones. 
Salvia, A. y Miranda, A. (2000). Sombras nada más. Transformaciones en las condiciones de vida de los jóvenes de los noventa (ponencia). Buenos Aires: CLACSO.

Salvia, A. y Tuñón, I. (2005). Una deuda social pendiente: la exclusión juvenil frente a las politicas fallidas de inclusión. Obtenido de Seminario Efectos Distributivos del gasto social en educación y formación de trabajadores: http://200.16.86.38/ uca/common/grupo32/files/salvia-exc-juvenil-2000-2005

Salvia, A., de Souza, D., Schmid, S., Scofienza, M. A. y van Raap, V. (2006). Los jóvenes pobres como objeto de politicas públicas ¿una oportunidad para la inclusión social o un derrotero de manipulación y frustraciones? (Ponencia). Tercer Congreso de Políticas Sociales, 18, 19 y 20 de octubre. Buenos Aires: Observatorio Social de la Universidad Católica de Buenos Aires. Buenos Aires.

Svampa, M. (2008). Cambio de época. Movimientos sociales y poder política. Buenos Aires: Siglo XXI Editores / CLACSO, Coediciones.

Vallés, M. (1999). Técnicas cualitativas de investigación social. Madrid: Síntesis. juvenil como efecto (inesperado) en la implementación de política pública destinada a artículo: jóvenes en la Argentina. Ánfora 20 (34), 15-36. Universidad Autónoma de Manizales. ISSN 0121-6538. 